\title{
Candida famata-induced fulminating cholecystitis
}

\section{Paulo Sergio Ramos de Araujo[1],[2], Zulma Medeiros ${ }^{[2]}$, Fabio Lopes de Melo ${ }^{[2],}$ Maria Amélia Maciel ${ }^{[1]}$ and Heloisa Ramos Lacerda de Melo ${ }^{[1]}$}

[1]. Serviço de Doenças Infecciosas, Hospital das Clínicas, Universidade Federal de Pernambuco, Recife, PE. [2]. Centro de Pesquisas Aggeu Magalhães, Fundação Oswaldo Cruz, Recife, PE.

\begin{abstract}
Lithiasic cholecystitis is classically associated with the presence of enterobacteria, such as Escherichia coli, Enterococcus, Klebsiella, and Enterobacter, in the gallbladder. Cholecystitis associated with fungal infections is a rare event related to underlying conditions such as diabetes mellitus, steroid use, and broad-spectrum antibiotic use for prolonged periods, as well as pancreatitis and surgery of the digestive tract. Here, we present the first reported case of a gallbladder infection caused by Candida famata.
\end{abstract}

Keywords: Cholecystitis. Candida. Gallbladder.

\section{INTRODUCTION}

Lithiasic cholecystitis, which predominantly occurs in the elderly, is classically associated with the presence of enterobacteria, such as Escherichia coli, Enterococcus, Klebsiella, and Enterobacter, in the gallbladder. Cholecystitis associated with fungal infections is a rare event related to underlying conditions such as diabetes mellitus, steroid use, and broad-spectrum antibiotic use for prolonged periods, as well as pancreatitis and surgery of the digestive tract ${ }^{1}$. Candida famata is mostly nonpathogenic in humans ${ }^{2}$. It has been isolated in patients with large burn areas ${ }^{3}$, stem cell transplant recipients ${ }^{4}$, individuals undergoing peritoneal dialysis ${ }^{5}$, patients with retinopathy ${ }^{6}$, and patients with acute infections of the central nervous system ${ }^{7}$.

\section{CASE REPORT}

Herein, we describe the case of a 79-year-old woman, born in Recife, State of Pernambuco, Brazil, who visited our hospital because of abdominal discomfort, nausea, and vomiting for 3 days. She did not experience any changes in her bowel habits. She had had diabetes for 2 years, for which she was irregularly taking metformin. She had no other comorbidities or fever, but she was dehydrated and dyspneic. Cardiopulmonary auscultation indicated normal sounds, and the abdominal

Address to: Dr. Paulo Sergio Ramos de Araujo. Depto de Medicina Tropical/ UFPE. Campus da UFPE. Av. Prof. Moraes Rego 1235, Cidade Universitária, 52670-901 Recife, PE, Brasil.

Phone: 5581 2126-3902.

e-mail: psergio@cpqam.fiocruz.br

Received 20 August 2013

Accepted 25 November 2013 examination indicated distension with bowel sounds. Lab oratory tests revealed hyperglycemia (glycemia $=400 \mathrm{mg} /$ $\mathrm{dL}$ ), neutrophil leukocytosis, impaired renal function (urea $=$ $83.0 \mathrm{mg} / \mathrm{dL}$ and creatinine $=2.1 \mathrm{mg} / \mathrm{dL}$ ), and a mild elevation in pancreatic enzyme levels. Urinalysis showed only mild proteinuria and glycosuria. Abdominal ultrasonography (USG) revealed thickening of the gallbladder wall and the presence of a calculus. No dilatation of the bile ducts was present, the pancreas had a normal texture, and no free liquid was present in the abdominal cavity. Blood and urine were collected for microbiological examination. Her clinical condition worsened with the aggravation of dyspnea and a decreased level of consciousness. She was admitted to the intensive care unit (ICU) for respiratory failure and hemodynamic instability, where she was provided with ventilatory support. Her hemodynamic condition deteriorated, and she was unresponsive to vigorous blood volume replacement.

The patient was administered a combination of meropenem and teicoplanin. She later presented with circulatory collapse, for which she received high doses of vasopressors. Her echocardiogram was normal. On her fifth day in the ICU, a new USG was performed to guide a percutaneous cholecystostomy, during which some pus-containing bile was drained. The drained material was cultured in 3 vials of blood using a medium for aerobic and anaerobic bacteria and fungi (Bact-Alert Bottles). The procedure was performed following the principles of safe surgery. However, after hemodialysis, her hemodynamic condition deteriorated again, and refractory metabolic acidosis occurred. The patient then died on the sixth day of hospitalization. Both blood and urine cultures were negative for pyogenic bacteria and fungi. Candida famata was identified in all 3 samples using the Vitek $2 \circledR$ automated system (degree of precision $>99 \%$ ), which is a rapid and accurate method for the identification of yeast species in clinical mycology laboratories, some of which are relatively uncommon ${ }^{8}$. 


\section{DIscussion}

Candidiasis often occurs in diabetic patients with inadequate glycemic control; however, this case is the first report of a gallbladder infection caused by Candida famata. We could not determine whether this infection was a primary or secondary infection of the gallbladder. The decompensation of her diabetes during hospitalization could have potentially favored a systemic infection by Candida famata, leading to a secondary infection of the gallbladder. Clinicians need to be aware of the possibility of this type of infection and judiciously administer antifungal agents in addition to surgical treatments in cases of biliary tract infection in diabetic patients, especially in those presenting with systemic clinical conditions.

\section{REFERENCES}

1. Morris AB, Sands ML, Shiraki M, Brown RB, Ryczak M. Gallbladder and biliary tract candidiasis: nine cases and review. Rev Infect Dis 1990; 12:483-489.
2. Desnos-Ollivier M, Ragon M, Robert V, Raoux D, Gantier JC, Dromer F. Debaryomyces hansenii (Candida famata), a rare human fungal pathogen often misidentified as Pichia guilliermondii (Candida guilliermondii). J Clin Microbiol 2008; 46:3237-3242.

3. Luo GX, Penq YZ, Nie ZH, Zhanq XB, Zhuanq Y, Yuan ZQ, et al. A clinical study of fungal infection in burn patients. Chinese $J$ Burns 2009; 25:91-93.

4. Wagner D, Sander A, Bertz H, Finke J, Kern WV. Breakthrough invasive infection due to Debaryomyces hansenii (teleomorph Candida famata) and Scopulariopsis brevicaulis in a stem cell transplant patient receiving liposomal amphotericin B and caspofungin for suspected aspergillosis. Infection 2005; 33:397-400.

5. Gupta A, Mi H, Wroe C, Jaques B, Talbot D. Fatal Candida famata peritonitis complicating sclerosing peritonitis in a peritoneal dialysis patient. Nephrol Dial Transplant 2006; 21:2036-2037.

6. Carrasco L, Ramos M, Galisteo R, Pisa D, Fresno M, Gonzalez ME. Isolation of Candida famata from a patient with acute zonal occult outer retinopathy. J Clin Microbiol 2005; 43:635-640.

7. Prinsloo B, Weldhagen GF, Blaine RW. Candida famata central nervous system infection. S Afr Med J 2003; 93:601-602.

8. Sanguinetti M, Porta R, Sali M, La Sorda M, Pecorini G, Fadda G, et al. Evaluation of VITEK 2 and RapID yeast plus systems for yeast species identification: experience at a large clinical microbiology laboratory. J Clin Microbiol 2007; 45:1343-1346. 